\title{
The wild grape genome sequence provides insights into the transition from dioecy to hermaphroditism during grape domestication
}

Hélène Badouin ${ }^{1 * \dagger}$, Amandine Velt $^{2 \dagger}$, François Gindraud ${ }^{1}$, Timothée Flutre ${ }^{3}$, Vincent Dumas ${ }^{2}$, Sonia Vautrin ${ }^{4}$, William Marande ${ }^{4}$, Jonathan Corbi ${ }^{1}$, Erika Sallet ${ }^{5}$, Jérémy Ganofsky ${ }^{1}$, Sylvain Santoni ${ }^{6}$, Dominique Guyot ${ }^{7}$, Eugenia Ricciardelli ${ }^{8}$, Kristen Jepsen ${ }^{8}$, Jos Käfer ${ }^{1}$, Hélène Berges ${ }^{4}$, Eric Duchêne ${ }^{2}$, Franck Picard ${ }^{1}$, Philippe Hugueney ${ }^{2}$, Raquel Tavares ${ }^{1}$, Roberto Bacilieri ${ }^{6^{*}+}$, Camille Rustenholz ${ }^{2^{*+}}$ and Gabriel A. B. Marais ${ }^{1{ }^{*}}$ (D)

\author{
* Correspondence: helene.badouin@ \\ univ-lyon1.fr; roberto.bacillieri@inrae. \\ fr; camille.rustenholz@inrae.fr; \\ gabriel.marais@univ-lyon1.fr \\ ${ }^{\dagger}$ Roberto Bacilieri, Camille \\ Rustenholz, and Gabriel AB Marais \\ have equivalent contribution as \\ senior authors. \\ Hélène Badouin and Amandine Velt \\ have equivalent contribution as first \\ authors. \\ 'Université de Lyon, Université Lyon \\ 1, CNRS, Laboratoire de Biométrie et \\ Biologie Evolutive UMR 5558 \\ F-69622 Villeurbanne, France \\ ${ }^{6}$ INRAE, UMR AGAP, Univ. \\ Montpellier, CIRAD, INRAE, Institut \\ Agro, Montpellier, France \\ ${ }^{2}$ Université de Strasbourg, INRAE, \\ SVQV UMR-A 1131, F-68000 Colmar, \\ France \\ Full list of author information is \\ available at the end of the article
}

\begin{abstract}
Background: A key step in domestication of the grapevine was the transition from separate sexes (dioecy) in wild Vitis vinifera ssp. sylvestris ( $V$. sylvestris) to hermaphroditism in cultivated Vitis vinifera ssp. sativa ( $V$. vinifera). It is known that $V$. sylvestris has an XY system and $V$. vinifera a modified $Y$ haplotype $(Y h)$ and that the sex locus is small, but it has not previously been precisely characterized.
\end{abstract}

Results: We generate a high-quality de novo reference genome for $V$. sylvestris, onto which we map whole-genome re-sequencing data of a cross to locate the sex locus. Assembly of the full $X, Y$, and $Y$ h haplotypes of $V$. sylvestris and $V$. vinifera sex locus and examining their gene content and expression profiles during flower development in wild and cultivated accessions show that truncation and deletion of tapetum and pollen development genes on the $X$ haplotype likely causes male sterility, while the upregulation of a $Y$ allele of a cytokinin regulator (APRT3) may cause female sterility. The downregulation of this cytokinin regulator in the Yh haplotype may be sufficient to trigger reversal to hermaphroditism. Molecular dating of $X$ and $Y$ haplotypes is consistent with the sex locus being as old as the Vitis genus, but the mechanism by which recombination was suppressed remains undetermined.

Conclusions: We describe the genomic and evolutionary characterization of the sex locus of cultivated and wild grapevine, providing a coherent model of sex determination in the latter and for transition from dioecy to hermaphroditism during domestication.

Keywords: Grapevine, Dioecy, Sex chromosomes, Sex-determining genes 


\section{Background}

Dioecy is rare in flowering plants $(\sim 6 \%)$ but over-represented among crops $(\sim 20 \%)$ [1]. In some cases, both wild and cultivated plants are dioecious (e.g., date palm, asparagus, persimmons). Other crops, such as grapevine, papaya, and strawberry, derive from dioecious progenitors and switched to hermaphroditism during domestication. The genes underlying this switch are currently not known in any crop. In Vitis vinifera ssp. sylvestris (V. sylvestris), the wild ancestor of domesticated and cultivated grapevine Vitis vinifera ssp. vinifera ( $V$. vinifera), wild females produce morphologically bisexual flowers, with retracted anthers that produce few and infertile pollen, while male flowers undergo early ovule abortion [2,3]. Old genetic studies suggested that in grape, sex is determined by one locus with three alleles M, F, and $\mathrm{H}$ [3]. In V. sylvestris, males are MF and females FF, and in $V$. vinifera, hermaphrodites can be $\mathrm{HF}$ or $\mathrm{HH}$ [2, 3]. A genetic analysis based on a population segregating for sex, obtained by crossing a $V$. vinifera genotype and an interspecific rootstock, allowed the identification of a 143-kb sex locus located on chromosome 2 in the $V$. vinifera reference genome [4]. However, this particular region is not well assembled in the reference genome (PN40024-version 12X.2) [5], the $\mathrm{F}$ and $\mathrm{H}$ haplotypes being mixed both on chr2 and on unassembled scaffold_233 [6]. Moreover, other genetic studies in V. vinifera found different size and boundaries for the sex locus (e.g., [7, 8]).

In $V$. sylvestris, a target sequencing in a core-collection of males and females using the genes previously identified in $V$. vinifera confirmed the sex-linkage of some of them, but not all [6]. It appeared that $V$. sylvestris has a XY system in which $\mathrm{M}=\mathrm{Y}$ and $\mathrm{F}=\mathrm{X}$. It was also found that the $\mathrm{H}$ haplotype of $V$. vinifera is likely a modified $\mathrm{Y}$ haplotype (hereafter called Yh) [6]. Differential expression analysis of male, female, and bisexual flowers in $V$. sylvestris and $V$. vinifera identified two candidate genes for female sterility, in particular APRT3, a putative cytokinin regulator located on chromosome 2 of $V$. vinifera [9], but no causative mutations have been identified. A genetic and evolutionary model to explain sex determination and switch to hermaphroditism in grapevine is currently lacking.

To build such a model, we aimed at (i) characterizing first the sex locus in $V$. sylvestris, the wild progenitor of $V$. vinifera, and (ii) comparing the sex locus in both wild and cultivated grapevine. We first produced a reference genome of $V$. sylvestris. We then re-sequenced a $V$. sylvestris cross and used a segregation analysis to identify sexlinked SNPs and locate the sex locus on the V. sylvestris genome. Comparing X, Y, and Yh haplotypes from both $V$. sylvestris and $V$. vinifera obtained by BAC sequencing, we characterized the presence/absence patterns in the sex locus genes. We then compiled the transcriptomic profiles of the sex locus genes during flower development. Combining all this information, we found a few candidate sex-determining genes and identified changes between the $\mathrm{Y}$ and $\mathrm{Yh}$ haplotypes that could explain the switch from dioecy to hermaphroditism during domestication.

\section{Results}

Sequencing and de novo assembly of a female $V$. sylvestris genome

For this work, we first produced a PacBio-based reference genome for $V$. sylvestris that was not available yet. We chose a female individual that was sequenced using SMRT- 
sequencing (120X). Contigs were assembled with falcon-unzip [10], and the grapevine reference genome (PN40024-version 12X.2, [5]) was used as a framework to help building pseudomolecules. We obtained a high-quality diploid assembly of $469 \mathrm{Mb}$ with a contig $\mathrm{N} 50$ of $1.7 \mathrm{Mb}, 98 \%$ of the gene content anchored on chromosomes and a BUSCO evaluation of 95\% (Table 1, Additional file 1: Table S1, Additional file 1: Fig. S1, Additional file 2), comparing favorably to other recently published plant genomes [11-13]. We annotated 39,031 protein-coding genes on primary contigs (Table 1).

\section{Location and boundaries of the sex locus}

On the newly obtained $V$. sylvestris assembly, the sex locus as described previously [4, 6] appeared to be fully included in the 5th largest contig of the assembly. To precisely identify the boundaries of the sex locus, a F1 family obtained from a controlled cross of $V$. sylvestris was generated and re-sequenced. Paired-end Illumina short reads of both parents and 10 offspring yielded 43 to 74 million of paired-end reads per individual (Additional file 1: Table S2). To overcome issues due to X-Y divergence, we used an iterative SNP-tolerant mapping procedure [14], eventually mapping $98.2 \%$ of reads in average (mapping coverage by individual 14x-28x, Additional file 1: Table S3-6).

On this data, single nucleotide polymorphisms (SNPs) that segregate with sex in our cross were identified with an empirical approach and with SEX-DETector ${ }^{++}$, a new version of the probabilistic method SEX-DETector that identifies sex-linked genes from patterns of segregation in a cross from RNA-seq data [15], which we further developed here to analyze DNA-seq data. As SNPs close to the sex locus might be genetically linked to the locus in a particular cross only, we used public whole-genome resequencing data to validate the sex-linked SNPs detected in this cross (Fig. 1a, b, Additional file 1: Tables S7-8). We searched for SNPs that were sex-linked in our cross and that were always heterozygous in males and homozygous in females in the validation dataset. We found that the $\mathrm{X}$ haplotype of the sex locus spanned $111 \mathrm{~kb}$ on the $V$. sylvestris chromosome $2(4,810,929$ to $4,921,949 \mathrm{bp}$, Fig. 1b, c, Additional file 1: Fig. S2-5), and we obtained a final dataset of 1865 XY SNPs (Additional file 3).

\section{Full assemblies of the $\mathrm{X}, \mathrm{Y}$, and $\mathrm{Yh}$ haplotypes}

To explore the differences in gene content and the structural rearrangements among haplotypes of the sex locus, we sequenced and assembled bacterial artificial chromosomes (BACs) covering the sex locus in a male of another $V$. sylvestris population and in $V$. vinifera cv. Cabernet-Sauvignon. We obtained full assemblies for the $\mathrm{X}$ and $\mathrm{Yh}$ haplotypes and an assembly with a 13-kb gap in the Y haplotype located in the VviPLATZy gene (see "Methods" section and Additional file 1: Text S1). When aligned, the $\mathrm{X}$ haplotype and two BAC contigs covering the $\mathrm{Y}$ haplotype showed colinearity (Fig. 2, Additional file 1: Fig. S2). When including also the flanking regions in the alignment, no inversion was found (Additional file 1: Fig. S6). To identify deletions, we used the alignment between the $\mathrm{X}$ and the $\mathrm{Y}$ haplotypes (Additional file 1: Fig. S2) as well as the mean mapping coverage in our re-sequencing dataset along the $\mathrm{X}$ haplotype, searching for two-fold reductions in males. This revealed eight regions spanning 500 to $5500 \mathrm{bp}$ and affecting one predicted gene (Additional file 1: Fig. S3). We detected more 


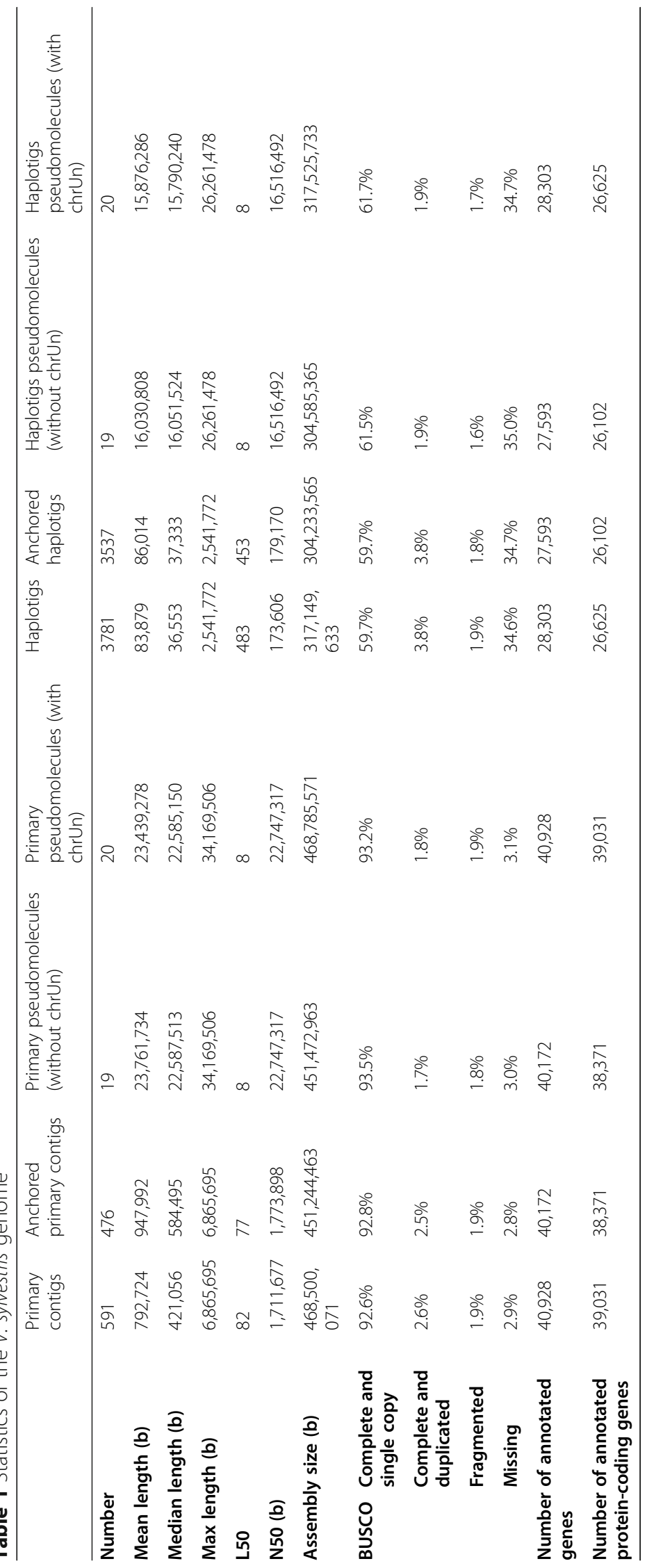




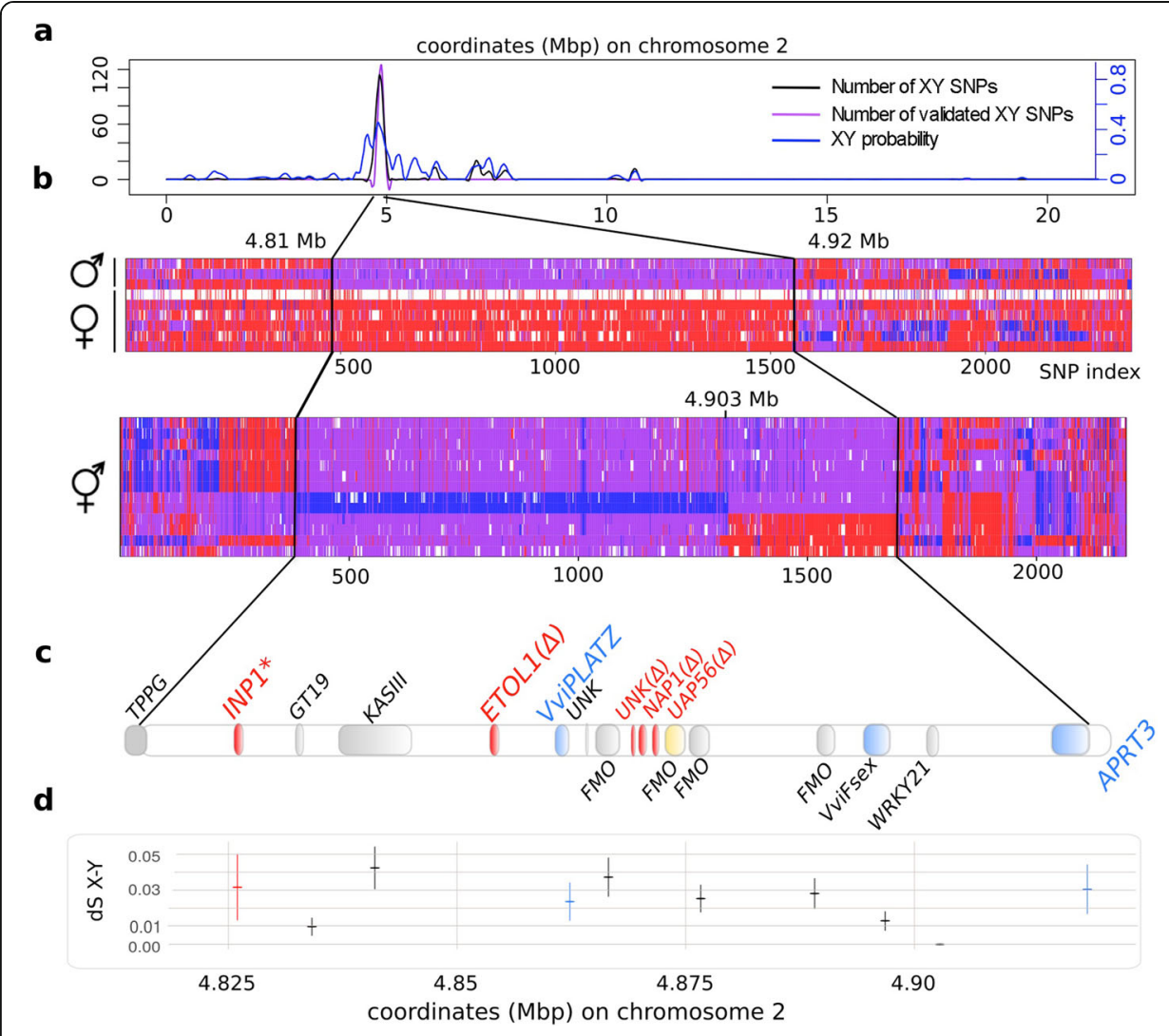

Fig. 1 Limit, gene content and synonymous divergence in the sex locus of $V$. sylvestris and $V$. vinifera. (a) Detection of sex-linked SNPs on chromosome 2. Left y axis: adjusted SNP number in $10 \mathrm{~kb}$ windows, black curve: XY single nucleotide polymorphisms detected by SEX-DETector++ in a cross of $\mathrm{V}$. sylvestris. Purple curve: candidate XY SNPs that show heterozygosity in males and homozygosity in females in a validation dataset of public whole-genome resequencing is drawn in purple; right $y$-axis (blue curve): adjusted mean posterior probability of being XY for SNPs in $10 \mathrm{~kb}$ windows. (b) Genotype of nine V. sylvestris individuals (top) and thirteen grapevine cultivars (bottom) in a validation dataset of public whole-genome re-sequencing, at locations of candidates XY SNPs. Red, purple, blue, and white marks represent $X X, X Y, Y Y$, and missing genotypes, respectively. In the cultivar panel, 11 cultivars are heterozygous (XY) for the sex-linked SNPs, with a Yh haplotype closely derived from Y (see text), while two cultivars (Chardonnay and Riesling) are homozygous YhYh. YhYh genotypes were previously found only in hermaphrodite cultivars [6]. The black lines highlight the limits of shared XY SNPs between the cross re-sequenced in the present study and the validation dataset. (c) Gene content and annotation and in the sex locus (approximate position). Genes highlighted in red are absent $(\Delta)$ or possess a frameshift deletion in the $X$ haplotype $\left(^{*}\right)$. Genes highlighted in blue are induced in males. The yellow gene is absent in the $Y$ haplotype. (d) Synonymous divergence between $X$ and $Y$ allele in the sex locus (+/- standard error), reflecting the age of recombination suppression. dS was only computed for genes present in both haplotypes

insertions of transposable elements in the $\mathrm{Y}$ haplotype than the $\mathrm{X}$ one (Table 2, Additional file 1: Fig. S2, Additional file 4, Additional file 5).

The comparison between the $\mathrm{X}, \mathrm{Y}$, and the $\mathrm{Yh}$ haplotypes showed strong similarities between $\mathrm{Y}$ and $\mathrm{Yh}$ haplotypes and confirmed that the $\mathrm{Yh}$ haplotype derives from the $\mathrm{Y}$ haplotype (Fig. 2, Additional file 1: Fig. S3 and S4). We however found that transposable elements' insertions are more numerous on the Yh haplotype compared to its $\mathrm{Y}$ counterpart (Table 2). Most of these insertions are located close to the APRT3 gene (Additional file 1: Fig. S4). Our assembly of the Yh haplotype was compared with published long-read-based sequences of $\mathrm{Yh}$ from Zinfandel [X-Yh] [19], Cabernet Sauvignon [X-Yh] [20], and Chardonnay [Yh-Yh'] [21]. Sequence identity was respectively 99.93\%, 99.97\%, and 99.62\%. Dot plots showed a full synteny between the Yh 


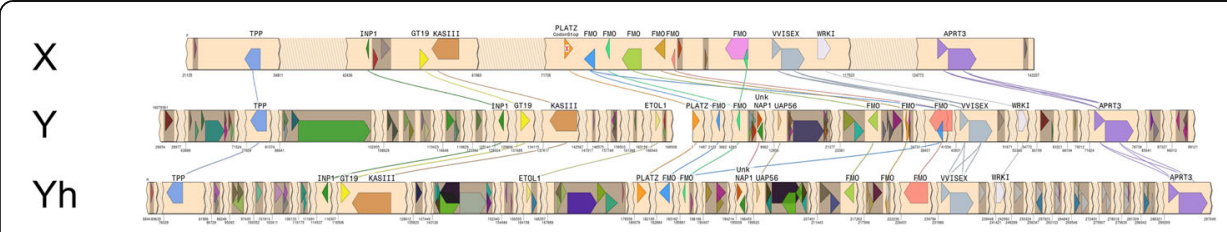

Fig. 2 Structural variation and gene content in the three haplotypes of the sex locus. (A) X haplotype of Vitis sylvestris; (B) Y haplotype of Vitis sylvestris; (C) Yh haplotype of the Cabernet Sauvignon cultivar. The gene positions were derived from a manually curated Eugene annotation [16]. The transposable elements (TEs) were detected with RepeatMasker 4.1 [17]; only TEs longer than 300 bp are represented (grayed rectangles). The synteny among sequences was obtained with SimpleSynteny [18] and orthologous genes connected by curved lines. The graphical representation is not drawn on scale: the distance between the TPP and APRT3 genes is 1.64 and 2.12 longer for the $Y$ and $Y h$ haplotypes respectively, as compared to the $X$ haplotype. Four genes (ETOL1, UAP56, NAP1, Unk) were detected only on the $Y$ and $Y$ h haplotypes

haplotypes. To explore the potential differences among $V$. vinifera cultivars, we investigated the SNP genotypes at the sex locus in publicly available DNA-seq data of 13 hermaphrodite grapevine cultivars [8] (Additional file 1: Table S9). Out of 13 cultivars, six harbored recombinant genotypes. The $5^{\prime}$ part of the sex locus was always either $\mathrm{XYh}$ or YhYh and spanned $93 \mathrm{~kb}(4.810-4.903 \mathrm{Mb})$ [6]. The remaining part of the sex locus (4.903-4.922 Mb) was either XX or XYh, but never YhYh (Fig. 1b, Additional file 1: Table S10). The genes essential for the male phenotype should therefore be located in the $93-\mathrm{kb}$ region of the sex locus.

\section{Age of the haplotypes}

To estimate the age of the sex locus, we calculated the synonymous divergence (dS) between $\mathrm{X}$ and $\mathrm{Y}$ coding DNA sequences (CDS) of XY gene pairs (Fig. 1c) as commonly done [22-24]. The dS values are all around 0.03 (except two genes for which $\mathrm{dS} X-\mathrm{Y} \leq$ 0.01 ), and the mean and median values for the sex locus are 0.024 and 0.026 respectively (Additional file 6). The homogeneity of the dS values suggests that the recombination was suppressed at about the same time for the whole locus. To obtain a rough divergence time from the $\mathrm{dS}$ values, we used a molecular clock $\left(7 \times 10^{-9}\right.$ base substitution/site/generation time) available for plants [25]. The average generation time in years is difficult to estimate in $V$. sylvestris as individuals are sexually mature at about 5 years old in the wild but become reproductively efficient later ( $>-10$ years old) and old

Table 2 Transposable element density in the $X, Y$ and $Y h$ haplotypes from the reference genome and the BACs

\begin{tabular}{lllll}
\hline Haplotype & DNA transposons & Retro-transposons & Other repeats & Total \\
\hline $\begin{array}{l}\text { V. sylvestris X } \\
\text { (ref. genome) }\end{array}$ & 23 & 23 & 9 & 55 \\
$\begin{array}{l}V . \text { sylvestris X } \\
\text { (BAC sequence) }\end{array}$ & 26 & 26 & 10 & 62 \\
$\begin{array}{l}V . \text { vinifera cv. Cabernet Sauvignon X } \\
\text { (BAC sequence) }\end{array}$ & 25 & 21 & 9 & 55 \\
$\begin{array}{l}\text { V. sylvestris } Y \\
\text { (BAC sequence) }\end{array}$ & 41 & 64 & 6 & 111 \\
$\begin{array}{l}V . \text { vinifera cv. Cabernet Sauvignon Yh } \\
\text { (BAC sequence) }\end{array}$ & 46 & 89 & 13 & 148 \\
\hline
\end{tabular}


individuals (some more than 100 years old) can be very large and productive [26]. Using a generation time of 5-10 years, we found that the sex locus could be 10.7-21.4 My old, setting a lower bound estimate. To get a higher bound estimate, we computed the average generation time from minimum and maximum age for sexual reproduction (52.5 years) and found that the sex locus could be 112.5 My old. We thus obtain a large range for the age of the sex locus, but it suggests that the sex locus is at least tens of million years old and is not recent.

We did a similar analysis for dating the $\mathrm{Yh}$ haplotype. As the $\mathrm{Yh}$ haplotype derives from the $\mathrm{Y}$ haplotype, we computed the $\mathrm{dS}$ values between $\mathrm{Yh}$ and $\mathrm{Y}$ alleles of the genes in the sex locus (Additional file 6). We found that the mean and the median $\mathrm{dS}$ are 0.0065 and 0 respectively, which are much lower values compared to what we obtained for the comparison between the $\mathrm{X}$ and $\mathrm{Y}$ haplotypes. Using the $\mathrm{dS} \mathrm{Y}-\mathrm{Yh}$ and using the minimum and average generation times mentioned above, we found that the Yh haplotype might be 2.32 to $24.4 \mathrm{MY}$ old. Using a corrected mean (after removal of two outliers with high dS values) dS Y-Yh instead, we found that Yh haplotype might be 0.57 to $6 \mathrm{MY}$ old. Our lower bound estimate is thus much higher than the estimated date of the domestication of grapevine.

\section{Finding candidate male-sterility genes}

The classical genetic models for sex determination in grapevine suggested that the sex locus contains two genes (with a dominant $S o=$ suppressing ovule allele for the first gene, and a recessive $s p=$ suppressing pollen for the second one, see [3]). According to these models, females are so sp/so $s p$ and males So Sp/so sp; the X haplotype should thus bear the $s p$ recessive allele and the $\mathrm{Y}$ haplotype the So dominant one. To identify candidates, we compared the gene content of X, Y, and Yh haplotypes (Fig. 3a) and searched for presence/absence patterns and loss-of-function mutations (Fig. 3b). We also mapped a public RNA-seq dataset of males, females, and hermaphrodites flower buds of $V$. sylvestris and $V$. vinifera at four developmental stages [27] (Additional file 1: Table S11), measuring the total (Fig. 4a) and the allele-specific (X, Y, and Yh, Fig. 4b) expression of transcripts.

First, we investigated the presence of $\mathrm{X}$ recessive mutations possibly causing male sterility. We found that the gene at one limit of the sex locus, annotated as INAPERTURATE (INP1) [21], showed a 8-bp deletion in exon2 in all $\mathrm{X}$ haplotypes, resulting in a premature stop codon in its DOG domain (involved in DNA binding) and a truncated protein (Fig. 3b). We also found that four genes were absent from the $\mathrm{X}$ haplotype (Figs. 1a, 2, and 3a, Additional file 1: Fig. S2). They included a gene previously annotated [6] as a short homolog of Ethylene-overproducer-like-1 (ETOL1). Three other genes were also absent from the $\mathrm{X}$ haplotype, namely a gene encoding a short peptide of unknown function (Unk), NAP1 and UAP56A.

\section{Finding candidate female-sterility genes}

Second, we searched for dominant mutations causing female sterility in the $\mathrm{Y}$ haplotype and for possible mechanisms of reversion to hermaphroditism. The Y-specific genes described above (INP1, ETOL1, UNK, and UAP56) are not expressed at the stage where ovule abortion occurs and are unlikely candidates for female sterility (Additional file 1: Fig. S7). Therefore, we searched for differential expression of $\mathrm{X}$ and $\mathrm{Y}$ alleles in $\mathrm{XY}$ gene pairs. 


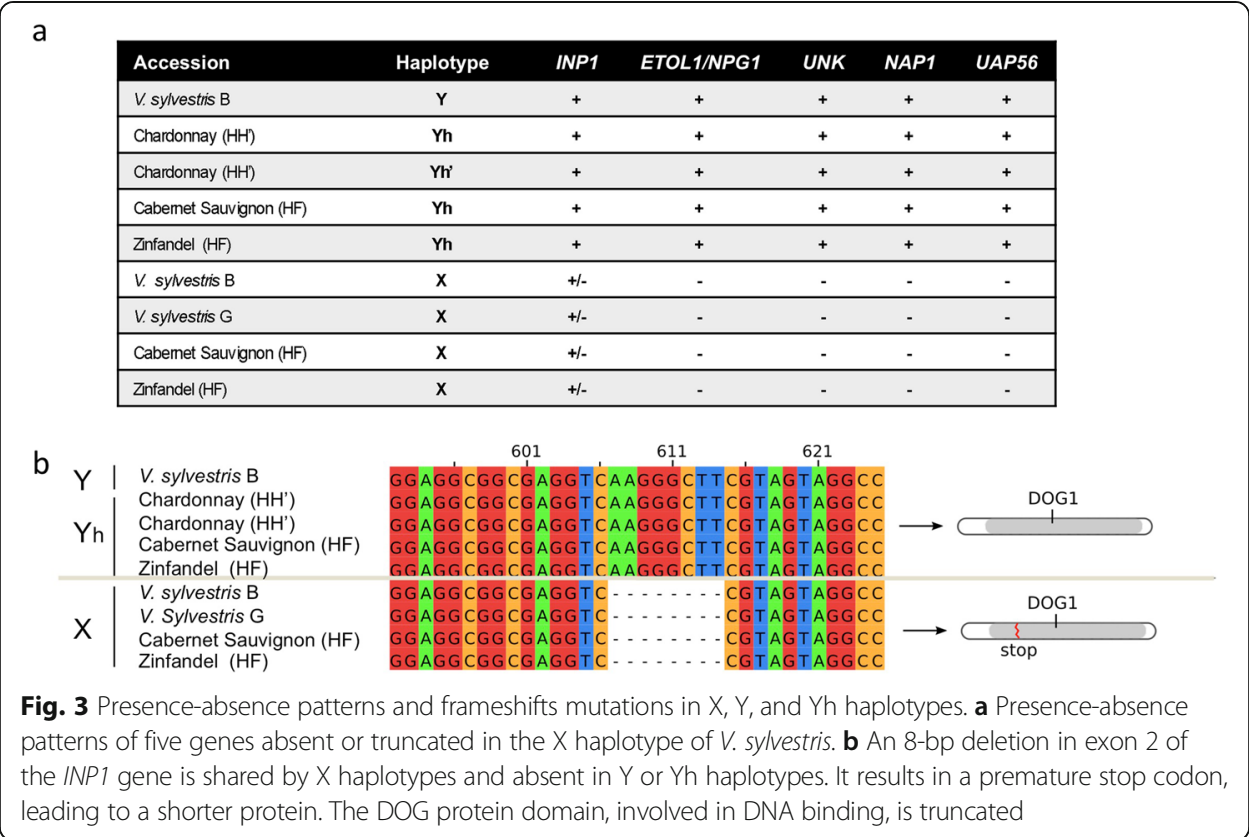

Three genes showed higher total expression in males than in females in the sex locus: $V v i-$ PLATZ, VviFSEX, and APRT3 (Fig. 4a). VviPLATZ and APRT3 showed a similar expression pattern in males, with a twofold induction during bud development. In females, APRT3 expression dropped down from early to late flower development. VviPLATZ expression was very low at all stages in females. Remarkably, only the Y alleles of VviPLATZ and APRT3 (VviPLATZy and APRT3y) showed a significant induction in male buds (Fig. 4b, Additional file 1: Fig. S8). VviFSEX showed a decreasing versus stable expression in female and males, respectively. Expression of the $\mathrm{X}$ and $\mathrm{Y}$ alleles were similar in males.

If these genes are involved in female sterility in males, they should have a modified expression in hermaphrodites. It is the case of all three genes (Fig. 4a). Even though VviPLATZy has a similar expression pattern in both hermaphrodites and males, it is expressed at a lower level all over the floral development in the former than in the latter (Fig. 4b). Both VviFSEX alleles show similar expression in hermaphrodites but contrary to males, their expression tend to decline during floral development similarly to females. In sharp contrast with males, APRT3y was not expressed in hermaphrodites, making it a particular interesting candidate female sterility gene. APRT3 is the ortholog of a gene encoding an enzyme of cytokinin elimination. We found that APRT3x is expressed both in female and male buds, especially at early stages, and is a ubiquitously expressed gene in grapevine (Additional file 1: Fig. S9). We found that all analyzed cultivars possessed at least one APRT3x allele (recombinant APRT3x/APRT3x genotypes were observed but no APRT3y/APRT3y, Fig. 1b).

\section{Discussion}

The wild grapevine genome and sex locus

Compared to the available V. vinifera reference genome (486 Mb, 2061 scaffolds, 14, 634 contigs with a N50 of $102 \mathrm{~kb}, 26,346$ genes and more than 41,000 proteins, see 


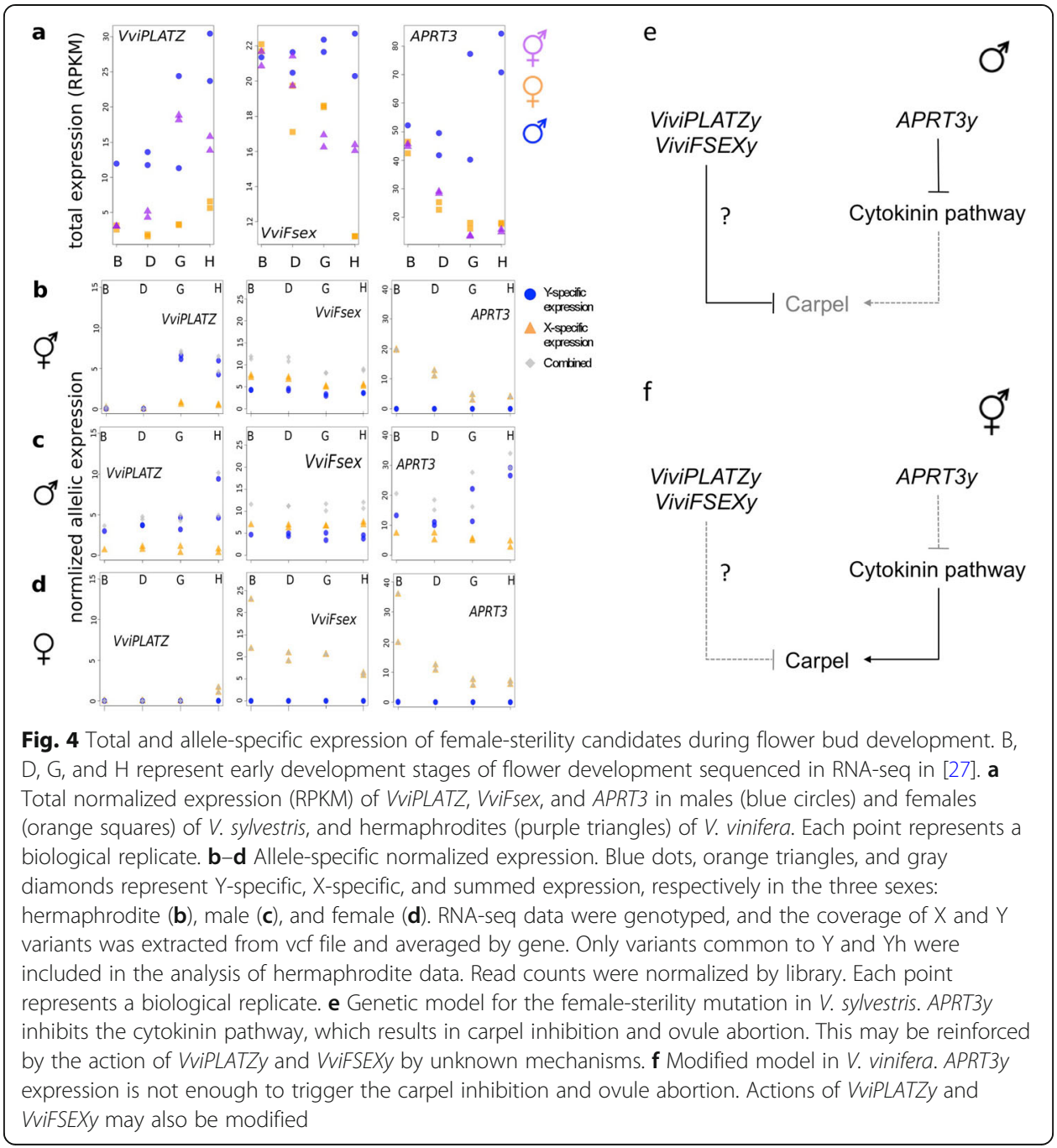

https://www.ncbi.nlm.nih.gov/genome/401?genome_assembly_id=214125), the $V$. sylvestris diploid reference genome that we generated here has similar size $(469 \mathrm{Mb})$, fewer contigs (591), and a larger N50 (1.7 Mb). Both genomes have a chromosomelevel assembly and are of high quality (Additional file 2).

In agreement with previous work in $V$. vinifera, we found that the sex locus is on chromosome 2 of $V$. sylvestris too. Our study shows that it is $111 \mathrm{~kb}$ long, smaller than the lower bound estimates from previous work. Picq et al. found that $Y A B B I$, a gene close to the sex locus, was not fully genetically linked to it [6]. This was confirmed here. The non-recombining region extends from TPP to APRT3; the genes outside of this region are recombining and are not part of the sex locus. They may be part of the sex determination gene network but not as master genes. Note that we use sex locus, sex-determining region, Ydifferentiated region, and non-recombining region of the sex chromosomes as synonyms here as in previous work in grapevine. The region that we delineated might however not include sex-determining genes only but also other genes unrelated to sex. 


\section{Molecular evolution of the grapevine sex locus}

We found that the divergence between the $\mathrm{X}$ and the $\mathrm{Y}$ haplotypes is low but using a molecular clock for plants and assuming a generation time from 5 to 52.5 years, we found that the sex locus may be between 10.7 and 112.5 MY old. These numbers may appear very high given the low $\mathrm{dS}$ we found for the sex locus. However, our results are consistent with the Vitis genus being slow-evolving [28, 29]. The radiation the Vitis genus (including subgenera Vitis and Muscadinia) from a dioecious ancestor took place 28 to $54 \mathrm{MY}$ ago [30, 31]. Previous work suggested that the $V$. sylvestris sex locus might be shared with other Vitis species [4, 6]. A recent study has identified the sex locus in several Vitis species and found it to be conserved in both subgenera [32]. If we assume that the sex locus is 28-54 MY old, as suggested by these observations, using our molecular clock, we obtain an average generation time of 13.1-25.2 years for $V$. sylvestris, which is not unrealistic. All these pieces of evidence converge to the idea that the grapevine sex locus is relatively old, at least as old as the Vitis genus itself and perhaps older.

Previous works have emphasized the importance of inversions (usually on the $\mathrm{Y}$ ) in suppressing recombination between sex chromosomes (e.g., [22, 24, 33]). Here we did not find evidence for inversions between the $\mathrm{X}, \mathrm{Y}$, and $\mathrm{Yh}$ haplotypes of the grapevine sex locus. Recombination must have been suppressed through another mechanism. Transposable elements are known to be abundant in non-recombining regions, which was also observed here in the $\mathrm{Y}$ haplotype of the $V$. sylvestris sex locus, but TE accumulation is usually seen as a consequence of the absence of recombination, not a cause [34, 35]. Unfortunately, recombination modifiers are poorly known in plants [36]. It will be interesting to explore this in future research.

\section{A genetic model for the sex determination of wild and cultivated grapevines}

In the classical genetic models, two genes with a dominant suppressing ovule allele ( $\mathrm{So}$ ) and a recessive suppressing pollen one $(s p)$, respectively, explain sex determination in grapevine [3]. When searching for $s p$, we identified five candidates: INP1, ETOL1, a short peptide of unknown function, NAP1, and UAP56A. INP1 loss-of-function mutant in Arabidopsis thaliana lacks pollen apertures [37], similarly to the pollen of female $V$. sylvestris [2,3]. We found that INP1 expression was highly specific of mature flower buds of $V$. vinifera, consistent with a role in late pollen development [38]. Inaperturate sterile pollen evolved independently at least six times in eudicots in association with dioecy [39]. It remains to be shown however that the absence of apertures in grapevine female pollen is sufficient to cause sterility in grapevine. Some of the A. thaliana INP1 knock-out mutants may be fertile [38], while those mutants in maize are infertile [40]. ETOL1 shows homology to NPG1, which is essential to pollen germination in A. thaliana [41], and the ethylene pathway has been shown to be determinant in floral morph determination in Cucumis melo [42]. NAP1 encodes a nucleosome assembly protein in A. thaliana. Two homologs of UAP56A, a DEAD-box ATP-dependent RNA helicase, have been shown to regulate programmed cell death during tapetum development in Oryza sativa [43], and disrupting them led to male sterility. None of these three genes were significantly expressed in the early developmental stages of the RNA-seq dataset that were produced to study ovule development (Additional file 1: Fig. S7), likely because male sterility occurs later than female 
sterility in $V$. sylvestris flowers [27]. INP1 and the four X-deleted genes were all present in at least one haplotype in cultivars. These results suggest that several recessive deletion mutations affecting genes involved in tapetum and pollen development may cause the male sterility syndrome (Fig. 5). We were not able to identify genes that could explain why the nonfunctional anthers observed in females are reflexed.

When searching for So, we identified three candidates: VviPLATZ, VviFSEX, and $A P R T 3$, a gene involved in the cytokinin pathway. Exogenous application of cytokinin is sufficient to restore female fertility in male V. sylvestris [44]. Previous in situ hybridization work suggested that APRT3 was induced during male bud development and absent in female buds [9]. We suggest that APRT3x performs essential functions in both $V$. sylvestris and $V$. vinifera. In contrast, APRT3y is specifically activated in males, suggesting that this allele form may trigger a decrease in cytokinin concentration in the carpel and cause its abortion (Figs. 4c, d and 5). Downregulation of VviPLATZy, APRT3y, and perhaps VviFSEX may be sufficient to cause reversal to hermaphroditism (Fig. 4, Additional file 1: Text S1). In addition (but not in a mutually exclusive way), transition to hermaphroditism may be caused by recombination upstream of the $A P R T 3$ promoter leading to the APRT3x/APRT3x genotype that is observed in several cultivars (Figs. 1b and 5).

We found a great functional coherence in $V$. sylvestris sex locus with tapetum and pollen genes involved in male-sterility and cytokinin pathway genes involved in femalesterility. Pollen and phytohormone pathway genes have previously been involved in sex determination $[45,46]$.

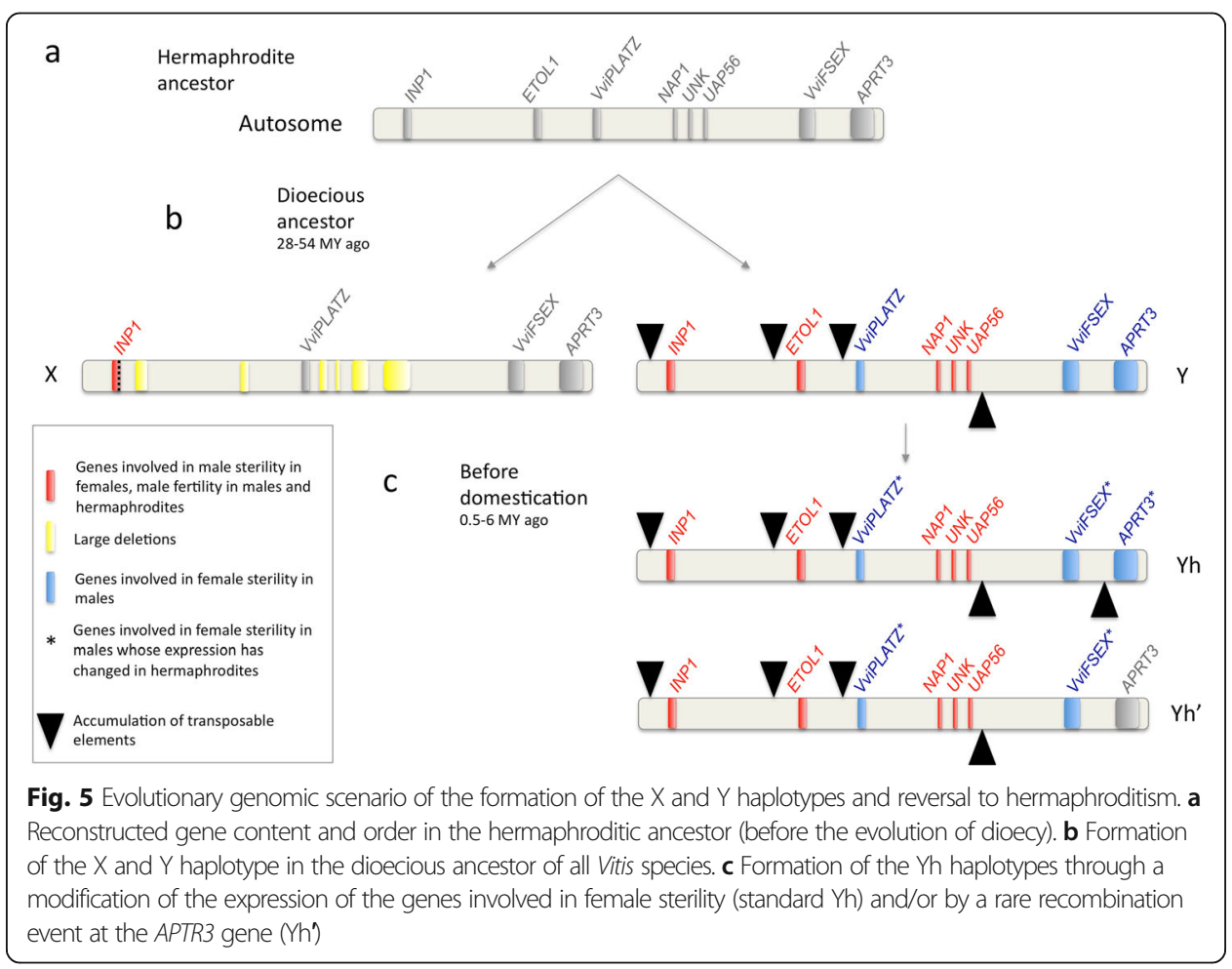




\section{Evolution of sex determination in plants: insights from grapevine}

The dominant theoretical model for the evolution of dioecy and sex chromosomes in plants relies on two sex-determining genes, carrying, one, a dominant female-sterility mutation and, the other, a recessive male-sterility mutation [47]. However, other models are possible $[45,46,48]$. In some species such as wild strawberries, Asparagus, date palms and kiwifruit, two candidate sex-determining genes have been found in agreement with the two-gene model [49-52], while in other species such as persimmons and possibly cucurbits, only one gene seems to be determining both male and female fertility [53-56].

Here we found several candidate sex-determining genes, which seems to rule out the single-gene model in grapevine. However, more than two candidates were identified, which suggest that the evolution of sex determination in grapevine might not have followed exactly the simple two-gene model in which a recessive malesterile mutation and a dominant female-sterile mutation successively spread in the population evolving towards dioecy. We identified several possible male-sterile mutations, and female-sterility is possibly mediated by several genes of the sex locus. Despite its small size, the sex locus is rather enriched in pollen and ovule genes, it is possible that sex determination initially evolved with two of these genes, and was reinforced later by secondary genes. However, the $\mathrm{X}$ and $\mathrm{Y}$ haplotypes' gene contents are conserved among Vitis and Muscadinia subgenera [4, 6, 32]. This suggests that all the sex-determining genes evolved in the dioecious ancestor of the whole Vitis genus (and possibly before).

Duplication and neofunctionalization have played important roles in the evolution of sex determination in all dioecious plants in which sex-determining genes have been identified [49-53]. In grapevine, we found that male sterility may have evolved through full or partial deletions as in other systems [49, 51, 52]. However, the evolution of the female sterility may have implied the expression divergence of the $\mathrm{Y}$ allele compared to the $\mathrm{X}$ allele of several genes (the $\mathrm{X}$ alleles conserving the ancestral function), a mechanism of neofunctionalization of the Y-allele, not documented before in dioecious plants.

How the expression of ViviPLATZy, VviFSEXy, and APRT3y was modified remains to be elucidated. Their cis-regulatory regions may have gained some new transcription factor binding sites [32]. Transposable elements may also have played a role. We indeed found accumulation of TEs close to ViviPLATZy, which may have influenced its expression patterns (Figs. 2 and 5). Remarkably, the most important accumulation of TEs in the Yh compared to its Y progenitor was upstream of the APRT3 gene, whose expression is strongly modified in hermaphrodite. TE insertions are known to lead to silencing of nearby genes, as in the case of the genes controlling sexual forms in monoecious cucurbits [57].

\section{Changes in the sex locus and grapevine domestication}

In hermaphrodites, ViviPLATZ, VviFSEX, and APRT3 expression is modified. The modification of APRT3 expression was the most drastic (Figs. 4 and 5), and it is possible that it is sufficient to restore female sterility. It is interesting to note that the main structural variation between $\mathrm{Y}$ and $\mathrm{Yh}$ also lies at APRT3. In one $\mathrm{Yh}$ haplotype, the APRT3 upstream region includes many TE insertions (as discussed 
above). Another Yh haplotype (Yh') found on Chardonnay [21] was formed by the recombination between a $\mathrm{Y}$ and a $\mathrm{X}$ at APRT3, which basically formed a $\mathrm{Y}$ haplotype with a APRT3x (Fig. 5). The Yh haplotypes are thus only slightly modified compared to the $\mathrm{Y}$ one. This may suggest that the switch to hermaphroditism during domestication was easy. This is in agreement with recent studies suggesting that dioecy is rare in flowering plants because it has a high reversion rate to hermaphroditism [58]. Hermaphrodites often occur naturally in dioecious plants [59]. Hermaphroditic $V$. sylvestris have already been observed in the wild [60] [3]. The Yh haplotypes may have pre-existed domestication as a standing natural variation. Our estimates of the age of the Yh haplotype (based on dS values) indeed support this idea. It is possible that the domestication process started with hermaphroditic individuals. It is also possible that the Yh haplotype was introgressed in the $\mathrm{V}$. vinifera (possibly several times independently) from $V$. sylvestris or other Vitis species late during the domestication process, a scenario that is consistent with the complex phylogenetic relationship between the $\mathrm{X}, \mathrm{Y}$, and $\mathrm{Yh}$ haplotypes from different cultivars and wild populations of $V$. sylvestris [6].

\section{Conclusions}

Our work provides a comprehensive characterization of the sex locus in $V$. sylvestris, a model for the sex determination in $V$. sylvestris and the switch to hermaphroditism in $V$. vinifera and a new reference genome, which is a valuable genomic resource to study diversity and domestication in grapevine. Future genetic and functional studies will be needed to dissect the role of the candidate genes that we found in different aspects of the male sterility syndrome, female sterility, and reversal to female fertility in hermaphrodites. It will be also interesting to understand better the evolution of the grapevine sex locus: how recombination was suppressed between the $\mathrm{X}$ and $\mathrm{Y}$ haplotypes. The study of the sex locus in many cultivated and wild grapevine populations may help understand more precisely the switch to hermaphroditism.

\section{Methods}

\section{Sequencing data}

Whole-genome sequencing (PacBio-long reads) of Sylvestris C1-2

The accession of Sylvestris C1-2 with plant code 8500.Col.C1-2, origin of the geographic location Sainte-Croix-en-Plaine, Haut-Rhin (68), France, was used for extraction of highmolecular-weight genomic DNA. The extraction was performed by the CNRGV (Centre National de Ressources Génomiques Végétales) - INRAE Toulouse. They started with $1 \mathrm{G}$ of frozen material $\left(-80^{\circ} \mathrm{C}\right)$, crushed it with liquid nitrogen, and used the Genomic-tip 100/G kit (Qiagen) for the extraction. The SMRT library preparation and sequencing on PacBio RSII platform (P6-C4 chemistry) was done by the IGM Genomics Center at the University of California, San Diego, following the standard PacBio protocols. A total of $129 \mathrm{X}$ coverage was obtained in order to perform de novo genome assembly.

\section{Whole-genome sequencing (Illumina-short reads) of Sylvestris C1-2}

DNA libraries with two different library size from leaves of Sylvestris C1-2 were prepared in order to perform $2 \times 151$ paired-end short reads sequencing (Illumina). One 
library had a library size of $740 \mathrm{bp}$ (860 bp with adapters), and the other library had a library size of $392 \mathrm{bp}$ (512 bp with adapters). The two libraries were sequenced on the same flowcell lane. The libraries preparation and Illumina sequencing was performed by the EPGV (Etude du Polymorphisme des Génomes Végétaux) - INRAE. A total of $338,109,086$ reads was obtained ( $\sim 100 \mathrm{X}$ coverage) for all samples together, used to polish the Sylvestris C1-2 PacBio assembly.

\section{RNA-sequencing of Sylvestris C1-2}

RNA-sequencing was performed on six samples of the Sylvestris C1-2 accession. Three biological replicates of whole green berries and three biological replicates of whole mid-ripening berries were sequenced. The RNA-seq library preparation was performed with the TruSeq Stranded mRNA Library Prep Kit (Illumina) and was sequenced in paired-end $(2 \times 100 \mathrm{bp})$ on HiSeq4000 platform (Illumina technology). Library preparation and sequencing was performed by the GenomEast platform-Strasbourg. A total of 1,113,531,260 reads were obtained for all samples together, used to assemble a Sylvestris transcriptome and perform genes annotation.

\section{Whole-genome re-sequencing of a cross between C1-5 (female Vitis sylvestris) and Martigny_2 (male Vitis sylvestris)}

These two accessions were crossed in INRAE Colmar ampelographic collection. The accession C1-5 female Vitis sylvestris (plant accession number at INRAE Colmar 8500.Col.C1-5) originated from the geographic location Sainte-Croix-en-Plaine, HautRhin (68), France, and the male accession Martigny_2 (plant accession number at INRAE Colmar 8500.Col.1) was an accession originating from the geographic location Martigny, Swiss. The progeny was grown at INRAE Colmar greenhouses. The DNA samples of both parents, of five male descendants (44613.Col.5026T, 44613.Col.5028T, 44613.Col.5029T, 44613.Col.5033T, and 44613.Col.5053T) and five female descendants (44613.Col.5035T, 44613.Col.5040T, 44613.Col.5046T, 44613.Col.5050T, and 44613.Col.5057 T), were sequenced. The quantity and quality of the DNA extracted from leaves (DNeasy plant mini kit, Qiagen) of 12 individuals from the cross (2 parents and 10 offspring) was checked prior to sequencing. Nanodrop analysis indicated that concentrations were always above $110 \mathrm{ng} \mu^{-1}$, and that more than $5 \mu \mathrm{g}$ DNA was available for all samples. Quality (fragment size) was checked using capillary electrophoresis (Fragment Analyzer) and was satisfactory. Based on quality check, we adapted sonication (using Covaris E220) in order to get mostly fragments of $250 \mathrm{bp}$. Twelve Illumina libraries were constructed and were pooled for two lanes of sequencing on an Illumina Hiseq 4000 machine in $2 \times 100$ bp paired-end mode. This yielded between 85 and 149 million reads per individual (Additional file 1: Table S2), which roughly corresponded to $17 \mathrm{X}$ to $30 \mathrm{X}$ coverage of re-seq data per individual.

\section{Genome assembly, cleaning, polishing, anchoring, and ordering}

Shortly, the Sylvestris C1-2 de novo genome assembly was performed with Falconintegrate (Falcon + Falcon-unzip), in order to obtain a diploid assembly (the two haplotypes). With Falcon, PacBio reads were self-corrected, assembly was performed, 
haplotypes were generated, assembly was polished with Arrow, and finally we obtained a phased diploid assembly of Sylvestris C1-2.

\section{Genome assembly and phasing}

The FALCON-integrate 1.8.4 tool used is available on github: https://github.com/PacificBiosciences/FALCON-integrate/tree/1.8.4. The FALCON parameters used for the Vitis sylvestris genome assembly are taken from the genome assembly of $V$. vinifera cv Cabernet Sauvignon paper [10] (pa_HPCdaligner_option $=-\mathrm{v}-$ dal128 -e0.75 -M60 -12500 -k18 -h1250 -s100 -w8; ovlp_HPCdaligner_option = -v -dal128 -M60 -e.96 -11500 -s100 -k24 -h1250; pa_DBsplit_option = -a -x500 -s200; ovlp_DBsplit_option = -s200; falcon_sense option = --output_multi --min_idt 0.70 --min_cov 4 --max_n_read 400 --n_core 8; falcon_sense_skip_contained $=$ False; overlap_filtering_setting $=$--max_diff 120 --max_cov 120 --min_cov 4 --bestn 10 --n_core 8). The phasing and haplotypes creation was performed with Falcon-unzip, with default parameters. The assembly was polished with FALCON and PacBio reads using Arrow (available in Falcon-integrate).

\section{Assembly polishing and finishing}

After the FALCON run, we performed additional polishing with Illumina reads. First, Illumina reads were aligned with bwa [61] mem and -M option on FALCON's genome assembly. Then, alignments were filtered with samtools to keep only primary alignments and concordant pairs. Finally, alignments were filtered with bamtools to keep alignments with an edit distance $\leq 5$. These filtered aligned reads are used to polish the assembly with the pacbio-util (version 0.2) from pacbio-utilities tool (https://github.com/douglasgscofield/PacBio-utilities). Then, the same alignment was performed on the genome polished with pacbio-utils, and this genome was polished with Illumina reads and PILON (v1.22 - https:/github.com/ broadinstitute/pilon). A few haplotigs may have remained in the primary contigs file. A tool is available, purge_haplotigs, to find these false primary contigs in order to move them to the haplotigs file. We used this tool to correct this and to finish our genome assembly (v1.0.4 - commit 6414f68 - https://bitbucket.org/mroachawri/ purge_haplotigs/src/master/).

\section{Quality control of the assembly}

Assembly statistics such as number of contigs, N50 and L50, were calculated with a home-made script. Genome assembly completeness was assessed with BUSCO [62] with the genome mode, the embryophyta_odb9 lineage, and the Arabidopsis species options (version 2.0 - https://gitlab.com/ezlab/busco). Nucmer tool (from MUMmer tool: https://sourceforge.net/projects/mummer/ - nucmer (version 3.1) parameters: -maxmatch $-1 \quad 100$-c 500) and the grapevine reference genome [5] (PN40024, version 12X.2 - https://urgi.versailles.inra.fr/Species/Vitis/Data-Sequences/Genome-sequences) were used to align the Sylvestris assembly to the PN40024 reference assembly in order to see completeness of this Sylvestris assembly. Nucmer and grapevine reference genome were also used to anchor and order Sylvestris contigs into chromosomes (with additional parameters $-r-q$ ), as it was done in the Chardonnay genome assembly paper [63]. 


\section{$B A C$ generation and sequencing}

Generation of BAC libraries of a Vitis sylvestris and Cabernet Sauvignon to obtain the sex locus $Y, Y h$, and $X$ sequences

We first chose a $V$. sylvestris male from a wild population spontaneously growing on a hill forest near Montpellier (France), on the Northern slope of the Pic Saint Loup mountain. Its male phenotype was confirmed over 5 years of observation of the flowers, both on the forest plant and on 5 of its clones planted in the INRAE Vassal Grape Collection in Marseillan, France (introduction name: Lambrusque PSL10; introduction number: 8500Mtp107). High molecular weight DNA was isolated from $40 \mathrm{~g}$ of PSL10 young leaves following the cell nuclei extraction method described in [64] with slight modifications [65]. The long DNA fragments were partially digested with EcoRI restriction enzyme, and fragments from 100 to $250 \mathrm{~kb}$ were selected. Sized and eluted DNA was then ligated into a pAGIBAC-EcoRI cloning vector and cloned into DH10B T1R Escherichia coli strain (Invitrogen). The resulting BAC clones were plated on a solid selective medium and organized in barcoded microplates using a robotic workstation QPix2 XT (Molecular Devices). The BAC library was named Vsy-B-Lamb. It consists of 27,648 clones of $113 \mathrm{~kb}$ size in average, representing a $6.4 \mathrm{x}$ genome equivalent coverage. The library was replicated for security reason, and the two copies were stored in separate freezers at $-80^{\circ} \mathrm{C}$.

We also used an existing BAC library of Cabernet Sauvignon (Cabernet Sauvignon clone 412, ENTAV-INRA ${ }^{\circledR}$, available at CNRGV Toulouse, France, named VVCSCabernetSauv. Both libraries are today available at CNRGV: https://cnrgv.toulouse. inrae.fr/fr/Banques/Vigne.

\section{BAC library screening}

The bacterial clones were deposited on a macroarray nylon membrane $(22 \times 22$ $\mathrm{cm}$ ), following a $6 \times 6$ grid pattern. Three copies of this gridded macroarray were created. To select BAC clones carrying the DNA fragments from the sex locus, we used specifically designed radio-labeled probes. These probes were defined using the sequences published in [6]: VSVV006, VSVV007, VSVV009, VSVV010, VSVV011 (GeneID GSVIVT01001275001, GSVIVT01001277001, GSVI VT01001286001, GSVIVT00007310001, GSVIVT00007312001 respectively). On the Vsy-B-Lamb BAC library, the hybridization of 3 separate pools of probes allowed to spot around 200 putatively positive BAC clones in total; the 30 clones showing the most intense spots were individually tested via real-time PCR using the same sequences used for probe design and 9 clones were validated. The same clustering of the 9 positive clones into two groups of alleles was obtained using two approaches: the first assignation based on their melting temperature curves similarities, and the second based on Sanger sequencing of internal and BAC-end sequence polymorphisms. These two groups correspond to the two alternate alleles expected in a "XY-like" sex region. Internal and BAC-ends sequences were also used to map the BACs on each other, for each allele, with the objective to sequence the minimal number of clones with an optimized overlapping to cover the whole region (minimum tilling paths composed of 3 and 4 clones respectively). The same procedure was used on the VVCS-CabernetSauv library to spot the sex- 
locus BACs and assign them respectively to the $\mathrm{X}$ and $\mathrm{Yh}$ allele, Yh designating modified Y haplotypes found in hermaphrodites.

\section{$B A C$ sequencing and assembly of $X, Y$, and $Y h$ haplotypes}

The sequences of the 7 sex-region BACs of $V$. sylvestris and the 6 BACs of Cabernet Sauvignon were obtained via a PacBio RSII sequencer (P6C4 chemistry). Sequencing was done in a pool of 20 individually tagged BAC clones. We used $2 \mu \mathrm{g}$ of each individual BAC clone DNA to prepare the PacBio SMRT $10 \mathrm{~kb}$ library. GeT-PlaGe Genomic Platform (INRAE-Toulouse, France) handled the sample loading on the RSII device and the data retrieval. We performed the detection and removal of residual $E$. coli sequences on raw reads. After a second cleaning step consisting in detecting and removing the vector sequences, individually tagged BAC sequences were assembled with the HGAP workflow (https://github.com/PacificBiosciences/Bioinformatics-Training/wiki/HGAP).

For each of the groups of BACs identified as above (X, Y, and Yh), the BAC sequences were joined using their overlapping, so to form long "haplotigs". For Vitis sylvestris, one haplotig was then assigned to the " $\mathrm{Y}$ haplotype" and the other to the " $\mathrm{X}$ haplotype" using the sex-discriminating polymorphisms described by [6], namely 10 SNPs for VSVV006, 7 SNPs for VSVV007, and 6 SNPs for VSVV009. These SNPs were found to be $100 \%$ associated with sex, in a worldwide collection of 22 males, 23 hermaphrodites, and 91 females [6], and we confirmed that the two haplotigs had either all male SNPs or all female SNPs. We used a similar procedure for calling X and Yh alleles from Cabernet Sauvignon BACs sequencing. Our Cabernet Sauvignon haplotig assemblies were also confirmed by comparison with those publicly available at that time, with a sequence identity of $99.97 \%$ over $260 \mathrm{~kb}$, the few differences being all located in mononucleotide repeats ([20], see http://169.237.73.197/CabSauv/).

\section{Genome and BAC annotation} Genome annotation

RNA Illumina paired-end reads obtained from grape berries of Sylvestris C1-2 (see above) were used to assemble a transcriptome in order to annotate the genome. Prinseq-lite [66], Ribopicker [67], and STAR [68] were used to clean the data, and the assembly was made using Trinity [69]. We assembled 398,189 putative transcripts (422,652,106 bp), with a N50 of $2217 \mathrm{bp}$, which roughly corresponds to the average size of Pinot noir transcripts (2056 bp). TransDecoder [69] detected 187,734 CDS with an average size of $832 \mathrm{bp}$ (of which 60, 985 CDS are larger than $832 \mathrm{bp}$ ). A BUSCO analysis detected 1237 genes, of which 1166 (94\%) are complete. EuGene-EP [16] (Eukaryote Pipeline - v1.4 - http://eugene.toulouse. inra.fr/) was used to perform genes annotation on the Sylvestris assembly. The protein databases used for the genes annotation were the TAIR10, swissprot, and uniprot plants databases. The transcriptomes used for the genes annotation were our Sylvestris transcriptome, 812 manual annotated genes, and transcript sequences of $V$. vinifera from NCBI - 201711-08. The configuration file with all the parameters used is available as supplementary data (egnep. Vvi.for_Sylvestris.cfg). Primary contigs and haplotigs were annotated separately, with the same parameters. Finally, we obtained two gene annotations, one for primary contigs and one for haplotigs, with different files: gene annotation in a gff3 file, gene/ $\mathrm{mrna} / \mathrm{cds} / \mathrm{ncrna} /$ protein sequences in separate fasta files, and some statistics per gene. 


\section{BAC annotation}

The BAC sequences' annotation ( $V$. sylvestris $\mathrm{Y}$ haplotype $\mathrm{P} 2, V$. sylvestris $\mathrm{Y}$ haplotype $\mathrm{P} 1, V$. sylvestris $\mathrm{X}$ haplotype, $V$. vinifera Cabernet Sauvignon $\mathrm{Yh}$ and $\mathrm{X}$ haplotype sequences and the annotation with EuGene [16] (Eukaryote Pipeline - v1.4 - http://eugene.toulouse.inra.fr/)) was started with the same parameters and reference files than for the whole-genome annotation. The protein databases used for the genes annotation were the TAIR10, swissprot, and uniprot plants databases. The transcriptomes used for the genes annotation were our Sylvestris transcriptome, 812 manual annotated genes, and transcript sequences of $V$. vinifera from NCBI - 2017-11-08. The configuration file with all the parameters used is available as supplementary data (egnep. Vvi.for_Sylvestris.cfg). Then, the annotations specific to BACs were extracted and used as final BACs' annotation. We did not run Eugene on the 5 BAC sequences alone because EuGene learns from data, and we did not want to introduce a bias due to the low number of BAC sequences. Annotation of both full and partial fragments of transposable elements was carried out on the same BAC sequences with RepeatMasker 4.1 [17]

\section{Development of SEX-DETector++}

We developed a new version of the SEX-DETector software that implements a probabilistic method to study SNPs segregation in a family, taking into account genotyping errors. SEX-DETector++ is coded in $\mathrm{C}++$ and uses additional algorithmic optimization to reduce the running time and memory usage by about two orders of magnitude compared to the original code. SEX-DETector++ thus allows convenient usage on large, genome-wide genotyping datasets for which the original code would require prohibitively large running times and memory. The underlying model is the same as in the original code, but new functionalities were added to deal with genomic data as an input of the method (in the vcf format). The code is publicly available at https://gitlab.in2p3.fr/sex-det-family/sex-detector-plusplus, where technical and installation details can also be found.

\section{Characterization of the sex-linked region in the $V$. sylvestris genome}

\section{SNP-tolerant mapping and SNP discovery from whole-genome re-sequencing data}

Divergence between X and Y alleles can prevent SNP discovery. To avoid this issue, we carried out an iterative SNP-tolerant mapping similarly to the procedure described in [14]. Raw reads of twelve individuals of the same family (2 parents and 5 descendants of each sex) were first mapped against the V. sylvestris genome with gsnap [70] v201807-04 (-m 0.1) in standard mode. At each iteration step, SNP calling was performed: variants were called with samtools mpileup v1.3.1 [71] followed with Varscan v2.4.3 [72] mpileup2snp (Min coverage 8, Min reads2 2, Min var. freq 0.2, Min avg. qual 15, $P$ value thresh 0.01). Additional filters were applied: minimum frequency of variants reads between 0.25 and 0.75 for heterozygote genotypes in individual samples, maximum coverage of twice mode of the Gaussian distribution for each sample, only bi-allelic SNPs, minor frequency variant higher than 0.05 , and maximum rate of missing data 0.2. A SNP database was built with gsnap utilities to perform a SNP-tolerant mapping at the next iteration. We carried out two iterations of SNP-tolerant mapping until the rate of discovery of new XY SNPs (see below) became low. Finally, a 4th step of SNP- 
tolerant mapping with more stringent parameters (-m 0.05, primary alignments only) was performed in order to reduce the rate of false-positive SNPs.

\section{Detection and validation of sex-linked single nucleotide polymorphisms}

In order to detect SNPs linked to the sex-determining region, we run SEX-DETector++. This yielded 4113 sex-linked SNPs (with a posterior probability high than 0.6 ), 90.5\% of them on chromosome 2 . As the sex-determining regions is assumed to be small $[4,6]$ and the low number of offspring in our cross gives us access to few recombination events, the sex-linked region in our analysis may appear larger than the actual sex-determining region (SNP close to the sex locus tend to be linked to sex in a particular cross). To determine the boundaries of the sex-determining region, independent public data were analyzed in order to identify shared XY SNPs between different populations. WG-reseq and RNA-seq data from wild and cultivated grapevines were mapped against the $V$. sylvestris genome with gsnap v2018-07-04; SNPs were called with varscan v2.4.3. We searched for candidate XY-SNPs that were heterozygote in all male samples and homozygote in all female samples of the validation dataset. Validated XY-SNPs spanned $111 \mathrm{~kb}$ on chromosome 2 (from 4,810,929 to 4,921,949 bp), which indicated the boundaries of the sex-determining region. In order to complement SEX-DETector++ analysis, we also performed an empirical one. We searched for SNPs that overlapped the sex-determining region and were homozygote in female individuals of the cross and heterozygote in male individuals, allowing two missing alleles. This empirical analysis retrieved all 1406 XY SNPs identified by SEX-DETector++ plus 459 additional ones (+32.6\%). This final dataset of 1865 XY SNPs was used for downstream analyses.

\section{Determination of the synonymous divergence between $X$ and $Y$ alleles in the sex-linked region}

The dataset of XY SNPs was used to build X and Y allelic pseudosequences in coding regions, using a custom python script to substitute reference positions by X or Y SNPs respectively in the genome in respect with strand, and to extract and concatenate coding DNA sequences for each gene of the sex locus. The yn00 program of the PAML suite was used to estimate synonymous divergence $(\mathrm{dS})$ with standard error estimation [73]. The same procedure was used to calculate the dS between the $\mathrm{Y} V$. sylvestris haplotype and the Yh haplotype of Cabernet Sauvignon.

\section{Structural characterization of the sex-linked region in the V. sylvestris genome}

Structural gene annotations in the sex-linked regions were extracted from Eugene (see annotation section). Predicted proteins were mapped against the NCBI nr database with BLASTP, and functional annotations were manually analyzed. The best hit in Arabidopsis thaliana was used to name the genes. Transposable elements were detected with Red [74] and RepeatMasker 4.1 [17].

\section{Integrative search for sex-determining genes in the sex locus}

\section{Comparison of gene and TE content in $X, Y$, and $Y h$ haplotypes}

In addition to the female whole genome of $V$. sylvestris, we used the assembled BAC sequences of $\mathrm{X}$ and $\mathrm{Y}$ haplotypes of $V$. sylvestris and $\mathrm{Yh}$ and $\mathrm{X}$ haplotypes of Cabernet- 
Sauvignon. BLASTN (ncbi-blast v2.2.3) were carried out between pairs of haplotypes (X-Y, X-Yh, Y-Yh, from whole-genome and from BACs). Whole-haplotype comparisons were visualized with CIRCOS [75] to identify presence/absence patterns. Genic sequences were extracted in all haplotypes using Eugene annotations and BLASTN, and aligned with mafft v7 online service [76] to identify structural differences and mutations. The sequences of the putative sex-determining candidates were blasted against assemblies of other cultivated grapevine genomes (Chardonnay, Cabernet-Sauvignon and Zinfandel) to assess their presence or absence.

\section{Total and allele-specific expression of sex-linked genes}

We retrieved raw reads from 23 public libraries of female, male, and hermaphrodite bud flowers' samples from $V$. sylvestris at four developmental stages [27]. Reads were mapped on the $V$. sylvestris genome with gsnap v2018-07-04 (-m 0.1), with a mode tolerant to XY SNPs. Read counts were obtained with htseq-count v0.10.0 [77] and normalized by computing the RPKM (reads per count per millions of reads mapped). To specifically measure the expression of X and $\mathrm{Y}$ alleles, we performed SNP calling (gsnap $-\mathrm{m} 0.05$, varscan max missing data 0.4 and minor allele frequency 0.05 ) and extracted the positions corresponding to XY SNPs located in CDS from the vcf file. For each gene and library, we read the number of reads mapping on reference and variant alleles (corresponding to $\mathrm{X}$ and $\mathrm{Y}$ alleles respectively) with bedtools intersect [78]. Reads numbers were summed by gene and normalized by the total number of reads in the variant file for the library and the length of transcripts.

\section{Organ-specific expression of sex-linked genes in V. vinifera}

We retrieved and mapped transcriptomic data of $V$. sylvestris and $V$. vinifera in several organs and conditions against the genome of $V$. sylvestris (berries; developing seeds; leaves under normal, drought, and pathogenic conditions; stem; early and mature flower buds; Additional file 1: Table S12). We measured the gene expression levels in the different conditions, and computed an index of organ-specificity (Tau) on normalized expression levels (log (read count per kilobase per millions of mapped reads)). The Tau specificity index ranges between 0 and 1 and typically display two modes near 0 and 1 indicating ubiquitous and organ-specific genes respectively [79].

\section{Statistical analyses and data visualization}

Unless stated otherwise, statistical analyses were carried out in R v3.4.4 (2018-03-15) [80]. Data visualization was performed with $\mathrm{R}$ or with CIRCOS v0.69-6 [75]. The structural variation among the $\mathrm{X}, \mathrm{Y}$, and $\mathrm{Yh}$ haplotypes was represented using SimpleSynteny 4.1 (Veltri et al. 2016; https://www.dveltri.com/simplesynteny/). Adeneget was used to visualize SNP data in R [81].

\section{Supplementary information}

Supplementary information accompanies this paper at https://doi.org/10.1186/s13059-020-02131-y.

Additional file 1: Figures S1 to S9, Tables S1 to S12, Text S1.

Additional file 2. Assembly statistics.

Additional file 3. List of $X / Y$ SNPS.

Additional file 4 Transposable element positions on the $X$ haplotype of the $V$. sylvestris genome. 
Additional file 5. Transposable element content of the different BAC-derived haplotypes.

Additional file 6. Pairwise dS values of $X$ vs $Y$ and $Y$ vs $Y h$ haplotypes.

Additional file 7. Review History.

\section{Acknowledgements}

This work benefitted from the computing facilities of the CC LBBE/PRABI. Whole-genome PacBio was conducted at the IGM Genomics Center, University of California, San Diego, La Jolla, CA. RNA-seq and cross re-sequencing were performed by the GenomEast platform, a member of the "France Génomique" consortium (ANR-10-INBS-0009). Sylvestris C1-2 Illumina re-sequencing was performed by the Etude du Polymorphisme des Génomes Végétaux (EPGV, INRAE, Evry) facility. We thank Guillaume Barnabé for his help with transcriptome assembly. We thank Jérôme Gouzy for help with curation of the trinity dataset used for gene annotation and Aline Muyle for providing information on the code of SEX-DETector perl, Gisèle Butterlin, and Angélique Ardiller for technical assistance and the UEAV experimental platform (Unité d'Expérimentation Agronomique et Viticole) for plant material maintenance at INRAE Colmar, and Jernej Jakše and Urban Kunej (Univ. Ljubljana) for their help with repeat annotation. The authors would like to acknowledge support of GeT-PlaGe Genomic Platform (INRAE-Toulouse, France, https://get.genotoulffr) for providing PacBio BAC sequence data.

Review history

The review history is available as Additional file 7.

\section{Peer review information}

Tim Sands was the primary editor of this article and managed its editorial process and peer review in collaboration with the rest of the editorial team.

\section{Authors' contributions}

GABM, PH, CR, ED, RT, and RB conceived the research. VD and ED obtained plant material and DNA for cross resequencing and genome sequencing. WM obtained DNA for whole-genome PacBio sequencing. TF and RB obtained plant material and DNA for BAC sequencing and with SS defined probes for BAC screening. SV and HBe performed BAC library production and screening, BAC sequencing, and assembly. AV, CR, and JC assembled the genome. RB and TF analyzed BAC sequences and assigned them to sex haplotyes. SS sequenced the gaps between BAC contigs. WM, $\mathrm{HBe}, \mathrm{ER}, \mathrm{K}$, and CR coordinated the sequencing of the genome. ED and CR coordinated the sequencing of the transcriptome. GABM coordinated the sequencing of the cross. JG and RT assembled the transcriptome. AV, CR, and ES annotated the genome and the BAC sequences. FG, FP, DG, JK, and GABM developed SEX-DETector++. HBa, AV, and CR analyzed RNA-seq data. HBa analyzed WGS data and performed structural and molecular evolution analysis of the sex locus. $H B a, C R$, and RB performed integrative analysis of sex determination. PH, RT, GABM, and RB funded the study. $\mathrm{CR}$, GABM, and RB coordinated the project. HBa and GABM wrote the manuscript, with contributions from all authors. The authors read and approved the final manuscript.

\section{Funding}

This work was supported by ANR grants ANR-13-BSV6-0010 to PH and ANR-14-CE19-0021-01 to GABM, an INRA starter grant BAP2014_44-SELVI to TF and UMT Géno-vigne ${ }^{\oplus}$ grant to RB.

\section{Availability of data and materials}

All raw reads, genome assembly, and gene annotation are available in ENA under the bioproject PRJEB37020 [82]. The source code of SEX-DETector++ is available at the url https://gitlab.in2p3.fr/sex-det-family/sex-detector-plusplus [83] available under the GNU General Public License v3.0 and archived at Zenodo [84].

\section{Ethics approval and consent to participate}

Not applicable.

\section{Consent for publication}

Not applicable.

\section{Competing interests}

The authors declare that they have no competing interests.

\section{Author details}

${ }^{1}$ Université de Lyon, Université Lyon 1, CNRS, Laboratoire de Biométrie et Biologie Evolutive UMR 5558, F-69622 Villeurbanne, France. ${ }^{2}$ Université de Strasbourg, INRAE, SVQV UMR-A 1131, F-68000 Colmar, France. ${ }^{3}$ GQE-Le Moulon, INRAE, Univ. Paris-Sud, CNRS, AgroParisTech, Univ. Paris-Saclay, 91190 Gif-sur-Yvette, France. ${ }^{4}$ INRAE, Centre National de Ressources Génomiques Végétales, F-31326 Castanet-Tolosan, France. ${ }^{5}$ LIPM, Université de Toulouse, INRAE, CNRS, Castanet-Tolosan, France. ${ }^{6}$ INRAE, UMR AGAP, Univ. Montpellier, CIRAD, INRAE, Institut Agro, Montpellier, France. ${ }^{7}$ PRABI, Université Lyon 1, F-69622 Villeurbanne, France. ${ }^{8} \mid$ GM Genomics Center, University of California, San Diego, La Jolla, CA, USA. 
Received: 3 March 2020 Accepted: 5 August 2020

Published online: 07 September 2020

\section{References}

1. Renner SS. The relative and absolute frequencies of angiosperm sexual systems: dioecy, monoecy, gynodioecy, and an updated online database. Am J Bot. 2014;101:1588-96.

2. This P, Lacombe T, Thomas MR. Historical origins and genetic diversity of wine grapes. Trends Genet. 2006;22:511-9.

3. Coito JL, Silva HG, Ramos MJ, Cunha J, Eiras-Dias J, Amâncio S, Costa MM, Rocheta M. Vitis flower types: from the wild to crop plants. PeerJ. 2019;7:e7879.

4. Fechter I, Hausmann L, Daum M, Sörensen TR, Viehöver P, Weisshaar B, Töpfer R. Candidate genes within a 143 kb region of the flower sex locus in Vitis. Mol Gen Genomics. 2012;287:247-59.

5. Canaguier A, Grimplet J, Di Gaspero G, Scalabrin S, Duchêne E, Choisne N, Mohellibi N, Guichard C, Rombauts S, Le Clainche I. A new version of the grapevine reference genome assembly (12X. v2) and of its annotation (VCost. v3). Genomics data. 2017;14:56.

6. Picq S, Santoni S, Lacombe T, Latreille M, Weber A, Ardisson M, Ivorra S, Maghradze D, Arroyo-Garcia R, Chatelet P. A small $X Y$ chromosomal region explains sex determination in wild dioecious $V$. vinifera and the reversal to hermaphroditism in domesticated grapevines. BMC plant biology. 2014;14:229.

7. Battilana J, Lorenzi S, Moreira FM, Moreno-Sanz P, Failla O, Emanuelli F, Grando MS. Linkage mapping and molecular diversity at the flower sex locus in wild and cultivated grapevine reveal a prominent SSR haplotype in hermaphrodite plants. Mol Biotechnol. 2013;54:1031-7.

8. Zhou Y, Massonnet M, Sanjak JS, Cantu D, Gaut BS. Evolutionary genomics of grape (Vitis vinifera ssp. vinifera) domestication. Proc Natl Acad Sci. 2017;114:11715-20.

9. Coito JL, Ramos MJ, Cunha J, Silva HG, Amâncio S, Costa MM, Rocheta M. VviAPRT3 and VviFSEX: two genes involved in sex specification able to distinguish different flower types in Vitis. Front Plant Sci. 2017:8:98.

10. Chin C-S, Peluso P, Sedlazeck FJ, Nattestad M, Concepcion GT, Clum A, Dunn C, O'Malley R, Figueroa-Balderas R, MoralesCruz A. Phased diploid genome assembly with single-molecule real-time sequencing. Nat Methods. 2016;13:1050.

11. Raymond O, Gouzy J, Just J, Badouin H, Verdenaud M, Lemainque A, Vergne P, Moja S, Choisne N, Pont C. The Rosa genome provides new insights into the domestication of modern roses. Nat Genet. 2018;50:772-7.

12. Badouin H, Gouzy J, Grassa CJ, Murat F, Staton SE, Cottret L, Lelandais-Brière C, Owens GL, Carrère S, Mayjonade B. The sunflower genome provides insights into oil metabolism, flowering and asterid evolution. Nature. 2017;546:148-52.

13. Belser C, Istace B, Denis E, Dubarry M, Baurens F-C, Falentin C, Genete M, Berrabah W, Chèvre A-M, Delourme R. Chromosome-scale assemblies of plant genomes using nanopore long reads and optical maps. Nature plants. 2018;4: 879-87.

14. Prentout D, Razumova O, Rhoné B, Badouin H, Henri H, Feng C, Käfer J, Karlov G, Marais GA. An efficient RNA-seq-based segregation analysis identifies the sex chromosomes of Cannabis sativa. Genome Res. 2020;30:164-72.

15. Muyle A, Kafer J, Zemp N, Mousset S, Picard F, Marais GA. SEX-DETector: a probabilistic approach to study sex chromosomes in non-model organisms. Genome Biol Evol. 2016;8:2530-43.

16. Sallet E, Gouzy J, Schiex T. EuGene: an automated integrative gene finder for eukaryotes and prokaryotes. New York: In Gene Prediction Springer; 2019: 97-120.

17. Smit A, Hubley R, Green P. RepeatMasker at. Nucleic Acids Res; 2016. http://repeatmasker.org

18. Veltri D, Wight MM, Crouch JA. SimpleSynteny: a web-based tool for visualization of microsynteny across multiple species. Nucleic Acids Res. 2016;44:W41-5.

19. Vondras AM, Minio A, Blanco-Ulate B, Figueroa-Balderas R, Penn MA, Zhou Y, Seymour D, Ye Z, Liang D, Espinoza LK. The genomic diversification of grapevine clones. BMC Genomics. 2019;20:972.

20. Minio A, Lin J, Gaut BS, Cantu D. How single molecule real-time sequencing and haplotype phasing have enabled reference-grade diploid genome assembly of wine grapes. Front Plant Sci. 2017;8:826.

21. Zhou Y, Minio A, Massonnet M, Solares E, LV Y, Beridze T, Cantu D, Gaut BS. The population genetics of structural variants in grapevine domestication. Nature plants. 2019;5:965-79.

22. Charlesworth D, Charlesworth B, Marais G. Steps in the evolution of heteromorphic sex chromosomes. Heredity. 2005;95: $118-28$.

23. Bergero R, Charlesworth D. The evolution of restricted recombination in sex chromosomes. Trends Ecol Evol. 2009;24: 94-102.

24. Muyle A, Shearn R, Marais GA. The evolution of sex chromosomes and dosage compensation in plants. Genome Biol Evol. 2017;9:627-45.

25. Ossowski S, Schneeberger K, Lucas-Lledó Jl, Warthmann N, Clark RM, Shaw RG, Weigel D, Lynch M. The rate and molecular spectrum of spontaneous mutations in Arabidopsis thaliana. Science. 2010;327:92-4.

26. Levadoux L. Les populations sauvages et cultivées des Vitis vinifera L. Institut national de la recherche agronomique; 1956.

27. Ramos MJN, Coito JL, Silva HG, Cunha J, Costa MMR, Rocheta M. Flower development and sex specification in wild grapevine. BMC Genomics. 2014;15:1095.

28. Jaillon O, Aury JM, Noel B, Policriti A, Clepet C, Casagrande A, Choisne N, Aubourg S, Vitulo N, Jubin C, et al. The grapevine genome sequence suggests ancestral hexaploidization in major angiosperm phyla. Nature. 2007:449:463-7.

29. Cenci A, Combes MC, Lashermes P. Differences in evolution rates among eudicotyledon species observed by analysis of protein divergence. J Hered. 2013;104:459-64.

30. Wan Y, Schwaninger HR, Baldo AM, Labate JA, Zhong G-Y, Simon CJ. A phylogenetic analysis of the grape genus (Vitis L. ) reveals broad reticulation and concurrent diversification during neogene and quaternary climate change. BMC Evol Biol. 2013;13:141.

31. Ma Z-Y, Wen J, Ickert-Bond SM, Nie Z-L, Chen L-Q, Liu X-Q. Phylogenomics, biogeography, and adaptive radiation of grapes. Mol Phylogenet Evol. 2018;129:258-67.

32. Massonnet M, Cochetel N, Minio A, Vondras AM, Lin J, Muyle A, Garcia JF, Zhou Y, Delledonne M, Riaz S, et al. The genetic basis of sex determination in grapes. Nat Commun. 2020;11:2902. 
33. Wang J, Na JK, Yu Q, Gschwend AR, Han J, Zeng F, Aryal R, VanBuren R, Murray JE, Zhang W, et al. Sequencing papaya X and Yh chromosomes reveals molecular basis of incipient sex chromosome evolution. Proc Natl Acad Sci U S A. 2012; 109:13710-5.

34. Vyskot B, Hobza R. The genomics of plant sex chromosomes. Plant Sci. 2015;236:126-35.

35. Tenaillon MI, Hollister JD, Gaut BS. A triptych of the evolution of plant transposable elements. Trends Plant Sci. 2010;15:471-8.

36. Lawrence EJ, Griffin CH, Henderson IR. Modification of meiotic recombination by natural variation in plants. J Exp Bot. 2017:68:5471-83

37. Dobritsa AA, Coerper D. The novel plant protein INAPERTURATE POLLEN1 marks distinct cellular domains and controls formation of apertures in the Arabidopsis pollen exine. Plant Cell. 2012;24:4452-64.

38. Dobritsa AA, Kirkpatrick AB, Reeder SH, Li P, Owen HA. Pollen aperture factor INP1 acts late in aperture formation by excluding specific membrane domains from exine deposition. Plant Physiol. 2018;176:326-39.

39. Furness CA. Why does some pollen lack apertures? A review of inaperturate pollen in eudicots. Bot J Linn Soc. 2007;155: 29-48.

40. Li P, Ben-Menni Schuler S, Reeder SH, Wang R, Suárez Santiago VN, Dobritsa AA. INP1 involvement in pollen aperture formation is evolutionarily conserved and may require species-specific partners. J Exp Bot. 2018;69:983-96.

41. Golovkin M, Reddy AS. A calmodulin-binding protein from Arabidopsis has an essential role in pollen germination. Proc Natl Acad Sci. 2003;100:10558-63.

42. Boualem A, Troadec C, Kovalski I, Sari MA, Perl-Treves R, Bendahmane A. A conserved ethylene biosynthesis enzyme leads to andromonoecy in two Cucumis species. PloS one. 2009;4(7):e6144.

43. Li X, Gao X, Wei Y, Deng L, Ouyang Y, Chen G, Li X, Zhang Q, WU C. Rice APOPTOSIS INHIBITOR5 coupled with two DEAD-box adenosine 5'-triphosphate-dependent RNA helicases regulates tapetum degeneration. Plant Cell. 2011;23: 1416-34.

44. Negi SS, Olmo HP. Sex conversion in a male Vitis vinifera L. by a kinin. Science. 1966;152:1624.

45. Fruchard C, Marais G. The evolution of sex determination in plants. Evol Dev Biol. 2017:1-14. https://link.springer.com/ referenceworkentry/10.1007\%2F978-3-319-33038-9_168-1.

46. Charlesworth D. Young sex chromosomes in plants and animals. New Phytol. 2019;224:1095-107.

47. Charlesworth B, Charlesworth D. A model for the evolution of dioecy and gynodioecy. Amer Nat. 1978;112:975-97.

48. Renner SS. Pathways for making unisexual flowers and unisexual plants: moving beyond the "two mutations linked on one chromosome" model. Am J Bot. 2016;103:587-9.

49. Tennessen JA, Wei N, Straub SC, Govindarajulu R, Liston A, Ashman T-L. Repeated translocation of a gene cassette drives sex-chromosome turnover in strawberries. PLoS Biol. 2018;16:e2006062.

50. Harkess A, Zhou J, Xu C, Bowers JE, Van der Hulst R, Ayyampalayam S, Mercati F, Riccardi P, McKain MR, Kakrana A. The asparagus genome sheds light on the origin and evolution of a young $Y$ chromosome. Nat Commun. 2017;8:1-10.

51. Torres MF, Mathew LS, Ahmed I, Al-Azwani IK, Krueger R, Rivera-Nuñez D, Mohamoud YA, Clark AG, Suhre K, Malek JA. Genus-wide sequencing supports a two-locus model for sex-determination in Phoenix. Nat Commun. 2018;9:1-9.

52. Akagi T, Pilkington SM, Varkonyi-Gasic E, Henry IM, Sugano SS, Sonoda M, Firl A, McNeilage MA, Douglas MJ, Wang T. Two Y-chromosome-encoded genes determine sex in kiwifruit. Nature Plants. 2019:5:801-9.

53. Akagi T, Henry IM, Tao R, Comai L. A Y-chromosome-encoded small RNA acts as a sex determinant in persimmons. Science. 2014;346:646-50.

54. Akagi T, Henry IM, Kawai T, Comai L, Tao R. Epigenetic regulation of the sex determination gene MeGI in polyploid persimmon. Plant Cell. 2016;28:2905-15.

55. Yang HW, Akagi T, Kawakatsu T, Tao R. Gene networks orchestrated by Me Gl: a single-factor mechanism underlying sex determination in persimmon. Plant J. 2019;98:97-111.

56. Boualem A, Troadec C, Camps C, Lemhemdi A, Morin H, Sari M-A, Fraenkel-Zagouri R, Kovalski I, Dogimont C, Perl-Treves R. A cucurbit androecy gene reveals how unisexual flowers develop and dioecy emerges. Science. 2015;350:688-91.

57. Martin A, Troadec C, Boualem A, Rajab M, Fernandez R, Morin H, Pitrat M, Dogimont C, Bendahmane A. A transposoninduced epigenetic change leads to sex determination in melon. Nature. 2009;461:1135-8.

58. Käfer J, Marais GA, Pannell JR. On the rarity of dioecy in flowering plants. Mol Ecol. 2017;26:1225-41.

59. Ehlers BK, Bataillon T. 'Inconstant males' and the maintenance of labile sex expression in subdioecious plants. New Phytol. 2007;174:194-211.

60. Negi S, Olmo H. Studies on sex conversion in male Vitis vinifera L.(sylvestris). Vitis. 1970;9:89-96.

61. Li H, Durbin R. Fast and accurate short read alignment with Burrows-Wheeler transform. Bioinformatics. 2009;25:1754-60

62. Simão FA, Waterhouse RM, loannidis P, Kriventseva EV, Zdobnov EM. BUSCO: assessing genome assembly and annotation completeness with single-copy orthologs. Bioinformatics. 2015;31:3210-2.

63. Roach MJ, Johnson DL, Bohlmann J, van Vuuren HJ, Jones SJ, Pretorius IS, Schmidt SA, Borneman AR. Population sequencing reveals clonal diversity and ancestral inbreeding in the grapevine cultivar Chardonnay. PLoS Genet. 2018;14: e1007807.

64. Peterson DG, Tomkins JP, Frisch DA, Wing RA, Paterson AH. Construction of plant bacterial artificial chromosome (BAC) libraries: an illustrated guide. J Agric Genomics. 2000;5:1-100.

65. Schweiger W, Steiner B, Vautrin S, Nussbaumer T, Siegwart G, Zamini M, Jungreithmeier F, Gratl V, Lemmens M, Mayer K. Suppressed recombination and unique candidate genes in the divergent haplotype encoding Fhb1, a major Fusarium head blight resistance locus in wheat. Theor Appl Genet. 2016;129:1607-23.

66. Schmieder R, Edwards R. Quality control and preprocessing of metagenomic datasets. Bioinformatics. 2011;27:863-4.

67. Schmieder R, Lim YW, Edwards R. Identification and removal of ribosomal RNA sequences from metatranscriptomes, Bioinformatics. 2012;28:433-5.

68. Dobin A, Davis CA, Schlesinger F, Drenkow J, Zaleski C, Jha S, Batut P, Chaisson M, Gingeras TR. STAR: ultrafast universal RNA-seq aligner. Bioinformatics. 2013;29:15-21.

69. Grabherr MG, Haas BJ, Yassour M, Levin JZ, Thompson DA, Amit I, Adiconis X, Fan L, Raychowdhury R, Zeng Q. Fulllength transcriptome assembly from RNA-Seq data without a reference genome. Nat Biotechnol. 2011;29:644.

70. Wu TD, Watanabe CK. GMAP: a genomic mapping and alignment program for mRNA and EST sequences. Bioinformatics. 2005;21:1859-75. 
71. Li H, Handsaker B, Wysoker A, Fennell T, Ruan J, Homer N, Marth G, Abecasis G, Durbin R. The sequence alignment/map format and SAMtools. Bioinformatics. 2009;25:2078-9.

72. Koboldt DC, Zhang Q, Larson DE, Shen D, McLellan MD, Lin L, Miller CA, Mardis ER, Ding L, Wilson RK. VarScan 2: somatic mutation and copy number alteration discovery in cancer by exome sequencing. Genome Res. 2012;22:568-76.

73. Yang Z. PAML 4: Phylogenetic Analysis by Maximum Likelihood. Mol Biol Evol. 2007;24:1586-91.

74. Girgis HZ. Red: an intelligent, rapid, accurate tool for detecting repeats de-novo on the genomic scale. BMC Bioinformatics. 2015;16:227.

75. Krzywinski M, Schein J, Birol I, Connors J, Gascoyne R, Horsman D, Jones SJ, Marra MA. Circos: an information aesthetic for comparative genomics. Genome Res. 2009;19:1639-45.

76. Katoh K, Rozewicki J, Yamada KD. MAFFT online service: multiple sequence alignment, interactive sequence choice and visualization. Brief Bioinform. 2019;20:1160-6.

77. Anders S, Pyl PT, Huber W. HTSeq - a Python framework to work with high-throughput sequencing data. Bioinformatics, 2015:31:166-9.

78. Quinlan AR, Hall IM. BEDTools: a flexible suite of utilities for comparing genomic features. Bioinformatics. 2010;26:841-2.

79. Yanai I, Benjamin H, Shmoish M, Chalifa-Caspi V, Shklar M, Ophir R, Bar-Even A, Horn-Saban S, Safran M, Domany E. Genome-wide midrange transcription profiles reveal expression level relationships in human tissue specification. Bioinformatics. 2005;21:650-9.

80. Team RC: R: a language and environment for statistical computing. 2013.

81. Jombart T. adegenet: a R package for the multivariate analysis of genetic markers. Bioinformatics. 2008;24:1403-5.

82. Badouin H, Velt A, Gindraud F, Flutre T, Dumas V, Vautrin S. et al. Raw and processed sequence data for Sylvestris C1-2 genome assembly, annotation and identification of the sex-determining region. European Nucleotide Archive. 2020. https://www.ebi.ac.uk/ena/browser/view/PRJEB37020. Accessed 17 August 2020.

83. Gindraud F, Guyot D, Käfer J, Picard F, Marais G. SEX-DETector++: an ultrafast version of SEX-DETector for studying sex chromosomes with genomic data. Github. 2020; https://gitlab.in2p3.fr/sex-det-family/sex-detector-plusplus. Accessed 4 Aug 2020

84. Gindraud F. Sex detector ++ source code. Zenodo. 2020; https://doi.org/10.5281/zenodo.3971106. Accessed: 4 Aug 2020.

\section{Publisher's Note}

Springer Nature remains neutral with regard to jurisdictional claims in published maps and institutional affiliations.

Ready to submit your research? Choose BMC and benefit from:

- fast, convenient online submission

- thorough peer review by experienced researchers in your field

- rapid publication on acceptance

- support for research data, including large and complex data types

- gold Open Access which fosters wider collaboration and increased citations

- maximum visibility for your research: over 100M website views per year

At $\mathrm{BMC}$, research is always in progress.

Learn more biomedcentral.com/submissions 